\title{
OPTIMAL DESIGN OF STIFFENED COMPOSITE UNDERWATER HULLS
}

\author{
Tanguy Messager $^{1}$, Pierre Chauchot ${ }^{2}$, Benoit Bigourdan ${ }^{2}$ \\ ${ }^{1}$ Institut de recherche en Génie civil et Mécanique (GeM), UMR CNRS 6183 \\ Ecole Centrale de Nantes, BP 92101, 44321 Nantes cédex3, France \\ tanguy.messager@univ-nantes.fr \\ ${ }^{2}$ Institut Français de Recherche pour l'Exploitation de la Mer (IFREMER) \\ Service Matériaux et Structures, BP 70, 29280 Plouzané, France \\ \{pierre.chauchot, benoit.bigourdan\}@ifremer.fr
}

Keywords: Composite, Cylinders, Stiffeners, Buckling, Optimization.

\begin{abstract}
This numerical study deals with the stiffened composite underwater vessel design. The structures under investigation are laminated cylinders with rigid end-closures and internal circumferential and longitudinal unidirectional composite stiffeners. Structural buckling induced by the high external hydrostatic pressure is considered as the major failure risk. An optimization design tool has been developed to obtain the reinforcement definition which maximizes the limit of stability: an analytical model of cylindrical composite shell buckling has been coupled to a genetic algorithm procedure. The numerical optimization tests carried out corroborate design tendencies validated previously by experiments.
\end{abstract}




\section{INTRODUCTION}

Numerous works have focused last decade on the design of deep submarine exploration housings and autonomous underwater vehicles [1-4]. For submersible devices of limited energy carrying capability, fiber reinforced composite materials enable low weight to displacement ratios and consequently enhance the endurance. The previous studies have shown that structural buckling induced by the high external hydrostatic pressure is the major risk factor under service conditions for deep underwater lengthy hulls.

In a previous work connected with IFREMER's (the French Research Institute for the Exploitation of the Sea) developments detailed in Refs.[5,6], the search of laminations of unstiffened composite cylinders that maximize the buckling pressure has been performed. A genetic algorithm procedure coupled with an analytical model of shell buckling has been developed to determine numerically optimized stacking sequences. Typical $\left[90_{N 1} / \Psi_{1} / \Phi_{N 2} / \Psi_{2} / 90_{N 3}\right]$ lamination patterns (the angles being measured with respect to the cylinder axis, the plies numbered from the inner to the outer surfaces of the shell, $\Psi_{1}$ and $\Psi_{2}$ denoting eventual transition zones and $\Phi$ being the minimum angle value) have been obtained both for carbon/epoxy and glass/epoxy tubes. The corresponding increases of buckling pressures, measured with respect to initial design solutions, have been verified by FEM calculus. Experimental buckling pressure values of thin glass/epoxy and carbon/epoxy cylinders, in good agreement with numerical results, have also demonstrated the significant gains due to the optimized laminations.

The present numerical study is devoted to the stiffened composite hull design. The structures studied and depicted in section §2 are lengthy laminated cylindrical shell with rigid endclosures and circumferential and longitudinal (called rings and stringers, respectively) unidirectional composite stiffeners. An optimization tool allowing the search of the reinforcement definition (lamination and stiffener characteristics) which maximizes the buckling pressure has therefore been developed by coupling an analytical stiffened shell buckling model (detailed in section §3) with a genetic algorithm procedure presented in section §4. The numerical tests performed in section $\S 5$ show substantial buckling pressure increases measured with respect to reference design solutions.

\section{STRUCTURES UNDER INVESTIGATION}

The overall geometry of the structures studied are lengthy composite cylinder having rigid end-closures on their ends [3,7]. As depicted in Fig.1, the geometry of the cylindrical laminated shell is characterized by its length $L$, its mean radius $R$ and its wall-thickness $h$ (see also Fig.2) composed of $N$ orthotropic cross-plies of equal thickness. Each composite ply number $i$ is assumed to be cross-ply of angle $\theta_{i}$ (i.e. made up of the same amounts of fibres in the $+\theta_{i}$ and $-\theta_{i}$ directions).

For the present work, the numerical investigations are based on numerical values related to IFREMER's investigations: the length is $L=400 \mathrm{~mm}$ and the internal diameter is $175 \mathrm{~mm}$. Three geometries of composite vessels have been investigated as reported in Tab.1. Each composite ply is assumed to be carbon fiber reinforced epoxy resin (T700 fibers, F100 resin) $0.625 \mathrm{~mm}$ thick and having the following orthotropic properties (modulus in GPa) [7]: $E_{1}=156 ; E_{2}=9.65 ; E_{3}=6.57 ; G_{12}=5.47 ; G_{13}=2.8 ; G_{23}=3.925 ; v_{12}=0.27 ; v_{13}=0.34$; $v_{23}=0.492$. At last, the cross-ply angles are limited to the following manufacturing set (values in degrees): $\theta_{k} \in\{30 ; 45 ; 60 ; 75 ; 90\}$. 


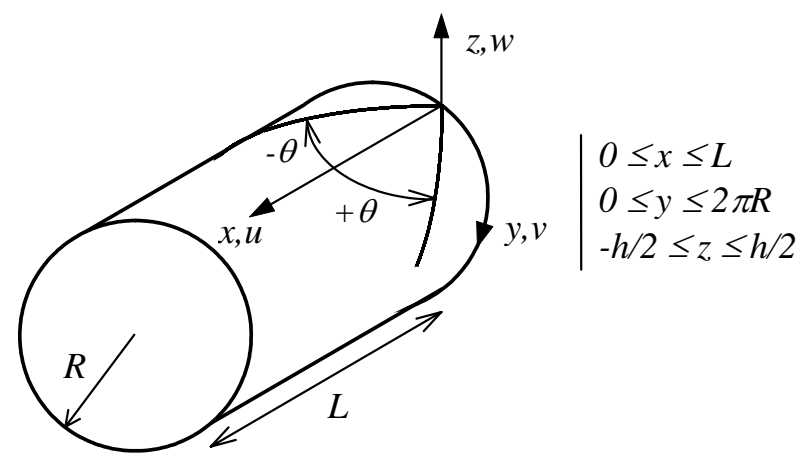

Figure 1: Cylindrical composite shell.

\begin{tabular}{llll}
\hline${\text { Cylinder } \mathbf{n}^{\circ}}^{\circ}$ & 1 & 2 & 3 \\
\hline Number of plies $\boldsymbol{N}$ & 10 & 16 & 26 \\
thickness $\boldsymbol{h}(\mathbf{m m})$ & 6.2 & 10.0 & 16.2 \\
mean radius $\boldsymbol{R}(\mathbf{m m})$ & 90.6 & 92.5 & 95.6 \\
Material volume $\left.\boldsymbol{V}_{\boldsymbol{c}} \mathbf{( 1 0}^{\mathbf{6}} \mathbf{m m}^{\mathbf{3}}\right)$ & 1.41 & 2.32 & 3.89 \\
\hline
\end{tabular}

Table 1: Geometries studied.

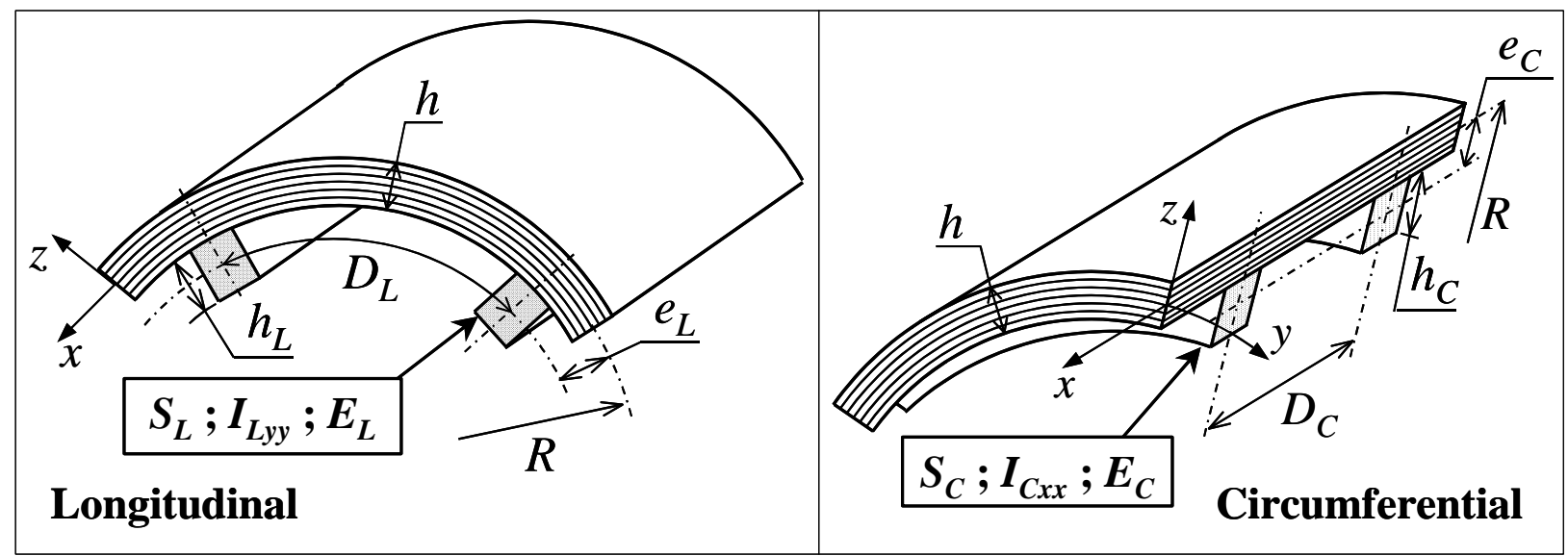

Figure 2: Stiffeners.

As schematized in Fig.2, the cylindrical shell is reinforced using $N_{L}$ and $N_{C}$ longitudinal and circumferential internal stiffeners, respectively. The corresponding cross-sections and eccentricities are denoted $S_{\alpha}$ and $e_{\alpha}$ (subscript $\alpha$ indicating the type of stiffener: $\alpha=L, C$ ). Following the works detailed in Refs.[8,9] and manufacturing requirements, the stiffeners are subjected to the following assumptions:

- The stiffeners are perfectly bounded to the shell.

- They are evenly distributed through the circumference and the length of the shell (see Fig.2):

$$
D_{L}=2 \pi\left(R-e_{L}\right) / N_{L} ; D_{C}=L / N_{C}
$$


- They are composed of unidirectional composite plies (of the same material of the shell) of $0^{\circ}$ angle with respect to the longitudinal direction of the stiffeners. Thus, the elastic modulus in this longitudinal direction of the stringers and the rings are done by:

$$
E_{L}=E_{C}=E_{1}
$$

- The stiffeners are loaded only by traction and bending (in the $(\vec{x}, \vec{y})$ plane) components, the torsional stiffness being neglected. The bending inertia modulus of the longitudinal and circumferential stiffeners are denoted $I_{L y y}$ and $I_{C x x}$ (in the $\vec{y}$ and $\vec{x}$ directions), respectively.

- They are sensitive to the shear transverse effects.

\section{ANALYTICAL STIFFENED COMPOSITE SHELL BUCKLING MODEL}

As detailed thereafter, the analytical modeling of the stiffened laminated shell buckling problem is treated similarly to the approach detailed in Ref.[6]. As shown by FE calculus and experimental result validations, such an approach has appeared to exhibit a good sensitivity to lamination parameters. The contribution of the stiffeners is here taken into account by correcting the overall laminate stiffness coefficients.

The developed buckling shell model is based on a Third Order Shear Deformable theory (TOSD). Hence, the displacement field is expressed as follows:

$$
\left\{\begin{array}{l}
U(x, y, z)=u+z \varphi_{x}-4 z^{3}\left(w_{, x}+\varphi_{x}\right) / 3 h^{2} \\
V(x, y, z)=v+z \varphi_{y}-4 z^{3}\left(w_{, y}+\varphi_{y}\right) / 3 h^{2} \\
W(x, y)=w
\end{array}\right.
$$

with $F_{, x}$ meaning $\partial F / \partial x$. Considering the cylindrical shell linear strain-displacement relations, it could be writen:

$$
\begin{aligned}
& \left\{\begin{array}{l}
\varepsilon_{x x} \\
\varepsilon_{y y} \\
2 \varepsilon_{x y}
\end{array}\right\}=\left\{\varepsilon_{m}\right\}+z\{\chi\}+z^{3}\left\{\chi^{a}\right\} \\
& =\left\{\begin{array}{l}
u_{, x} \\
v_{, y}+w / R \\
u_{, y}+v_{, x}
\end{array}\right\}+z\left\{\begin{array}{l}
\varphi_{x, x} \\
\varphi_{y, y} \\
v_{, x} / R+\varphi_{x, y}+\varphi_{y, x}
\end{array}\right\}-\frac{4 z^{3}}{3 h^{3}}\left\{\begin{array}{l}
w_{, x x}+\varphi_{x, x} \\
w_{, y y}+\varphi_{y, y}-v_{, y} / R \\
2 w_{, x y}+\varphi_{x, y}+\varphi_{y, x}-v_{, x} / R
\end{array}\right\} \\
& \text { and }\left\{\begin{array}{l}
2 \varepsilon_{y z} \\
2 \varepsilon_{x z}
\end{array}\right\}=\left(1-\frac{4 z^{2}}{h^{2}}\right)\{\gamma\}=\left(1-\frac{4 z^{2}}{h^{2}}\right)\left\{\begin{array}{l}
w_{, y}+\varphi_{y}-v / R \\
w_{, x}+\varphi_{x}
\end{array}\right\}
\end{aligned}
$$

Considering the hypothesis related to the stiffeners, the following tensile force and moment resultant components (expressed with respect to the mean-surface of the shell) are expressed as follows:

$$
\left\{\begin{array}{l}
N_{x} \\
M_{x}
\end{array}\right\}=\int_{-h / 2-h_{L}}^{h / 2}\left\{\begin{array}{c}
\sigma_{x x} \\
z \sigma_{x x}
\end{array}\right\} \mathrm{d} z ;\left\{\begin{array}{c}
N_{y} \\
M_{y}
\end{array}\right\}=\int_{-h / 2-h_{C}}^{h / 2}\left\{\begin{array}{c}
\sigma_{y y} \\
z \sigma_{y y}
\end{array}\right\} \mathrm{d} z
$$


where $h_{L}$ and $h_{C}$ are the total height of the stiffeners as depicted in Fig.2. The other terms are not dependant of the stiffeners and are given by:

$$
\left\{\begin{array}{c}
N_{x y}=N_{y x} \\
T_{x} \\
T_{y} \\
M_{x y}=M_{y x}
\end{array}\right\}=\int_{-h / 2}^{h / 2}\left\{\begin{array}{c}
\sigma_{x y} \\
\sigma_{x z} \\
\sigma_{y z} \\
z \sigma_{x y}
\end{array}\right\} \mathrm{d} z
$$

In that way, and considering the elastic orthotropic constitutive law of the composite crossplies, these resultants components are done by:

$$
\left\{\begin{array}{l}
\{N\} \\
\{M\} \\
\{T\}
\end{array}\right\}=\left[\begin{array}{cccc}
{[A]+[\hat{A}]} & {[B]+[\hat{B}]} & {\left[B^{a}\right]+\left[\hat{B}^{a}\right]} & 0 \\
{[B]+[\hat{B}]} & {[D]+[\hat{D}]} & {\left[D^{a}\right]+\left[\hat{D}^{a}\right]} & 0 \\
0 & 0 & 0 & {[E]}
\end{array}\right]\left\{\begin{array}{c}
\left\{\varepsilon_{m}\right\} \\
\{\chi\} \\
\{\chi \\
\{\gamma\} \\
\gamma\}
\end{array}\right\}
$$

using the following laminate stiffness coefficients [MES02]:

$$
\begin{gathered}
A_{i j}=\sum_{k=1}^{N} C_{i j}^{(k)}\left(z_{k}-z_{k-1}\right) ; B_{i j}=\frac{1}{2} \sum_{k=1}^{N} C_{i j}^{(k)}\left(z_{k}^{2}-z_{k-1}^{2}\right) ; D_{i j}=\frac{1}{3} \sum_{k=1}^{N} C_{i j}^{(k)}\left(z_{k}^{3}-z_{k-1}^{3}\right) ; \\
B_{i j}^{a}=\frac{1}{4} \sum_{k=1}^{N} C_{i j}^{(k)}\left(z_{k}^{4}-z_{k-1}^{4}\right) ; D_{i j}^{a}=\frac{1}{5} \sum_{k=1}^{N} C_{i j}^{(k)}\left(z_{k}^{5}-z_{k-1}^{5}\right) ; E_{i j}=A_{i j}-\frac{4}{h^{2}} D_{i j}
\end{gathered}
$$

and the additional terms due to the stiffeners:

$$
\left\{\begin{array}{l}
\hat{A}_{11} \\
\hat{B}_{11} \\
\hat{B}_{11}^{a} \\
\hat{D}_{11} \\
\hat{D}_{11}^{a}
\end{array}\right\}=\frac{E_{L}}{D_{L}}\left\{\begin{array}{l}
S_{L} \\
-e_{L} S_{L} \\
-e_{L}{ }^{3} S_{L}-3 e_{L} I_{L y y} \\
e_{L}{ }^{2} S_{L}+I_{L y y} \\
e_{L}{ }^{4} S_{L}+6 e_{L}{ }^{2} I_{L y y}
\end{array}\right\} ;\left\{\begin{array}{l}
\hat{A}_{22} \\
\hat{B}_{22} \\
\hat{B}_{22}^{a} \\
\hat{D}_{22} \\
\hat{D}_{22}^{a}
\end{array}\right\}=\frac{E_{L}}{D_{L}}\left\{\begin{array}{l}
S_{C} \\
-e_{C} S_{C} \\
-e_{C}{ }^{3} S_{C}-3 e_{C} I_{C x x} \\
e_{C}{ }^{2} S_{C}+I_{C x x} \\
e_{C}{ }^{4} S_{C}+6 e_{C}{ }^{2} I_{C x x}
\end{array}\right\}
$$

the other superscript ${ }^{\wedge}$ coefficients being zero. The governing equations of equilibrium are done by:

$$
\left\{\begin{array}{l}
N_{x, x}+N_{y x, y}=0 \\
N_{x, x}+N_{x y, x}+T_{y} / R=0 \\
T_{x, x}+T_{y, y}-N_{y} / R=0 \\
M_{x, x}+M_{y x, y}-T_{x}+P R\left(U_{, z}-W_{, x}\right) / 4=0 \\
M_{y, y}+M_{x y, x}-T_{y}+P R\left(V_{, z}-W_{, y}+V / R\right) / 2=0
\end{array}\right.
$$

where $P$ is the external hydrostatic pressure.

The displacement approximation functions satisfying the kinematic boundary conditions are chosen to be harmonic approximation functions defined as follows [5]: 


$$
\left\{\begin{array}{l}
u=u(x, y)=a_{u} \cos (m \pi x / L) \cos (n y / R) \\
v=v(x, y)=a_{v} \sin (m \pi x / L) \sin (n y / R) \\
w=w(x, y)=a_{w} \sin (m \pi x / L) \cos (n y / R) \\
\varphi_{\mathrm{x}}=\varphi_{\mathrm{x}}(x, y)=a_{\varphi x} \cos (m \pi x / L) \cos (n y / R) \\
\varphi_{\mathrm{y}}=\varphi_{\mathrm{y}}(x, y)=a_{\varphi y} \sin (m \pi x / L) \sin (n y / R)
\end{array}\right.
$$

where $m$ and $n$ are the numbers of longitudinal and circumferential half-waves of the buckling modes. Finally, after substituting the displacement approximations in Eq.(4), the governing relations (10) expressed using the resultants done by Eqs.(5,6) lead to the eigen-value buckling problem that can be expressed under a matrix form. As shown in Ref.[6], it can then be easily computed to estimate the buckling pressure $P_{c r}$.

\section{OPTIMIZATION PROCEDURE}

The optimization problems studied in section §5 implies several type of discrete parameters (standard angles of laminations, number of composite plies, numbers of rings and stringers). Hence it requires adequate methods dedicated to discontinuous design space analysis. Moreover, some previous works focusing on the optimal laminations of composite structures subjected to buckling have shown that these problems involve local optima, non-convexity, and then requires global optimization procedures $[5,8]$.

The numerous engineering design problems explored in recent years $[10,11]$ have demonstrated the usefulness and robustness of the genetic algorithms. The main principle is based on an analogy with natural evolution and biologically inspired operators: it manipulates a fixed number of potential design solutions (called individuals) and the fittest ones (leading to the best objective function $F$ values) are likely to survive, to recombine their features and thus to form a renewed population.

According to results taken from the literature and preliminary tests [5], the computed genetic algorithm manipulates directly integer parameters. The starting population is randomly created. The tournament selection, the whole arithmetical crossover and the random uniform mutation are applied. The foremost scheme, the genetic operators (also detailed in Refs.[5,10]) and the corresponding probability values are reminded in Fig.3. The GA finally sends back the best individual found. For the numerical applications performed, the numbers of individuals and generations have been chosen accordingly to the cardinalities of the optimization problems.

\section{NUMERICAL RESULTS}

In the next paragraphs, the unstiffened cylinders with the optimized laminations $[\theta]_{\text {Opti }}$ (obtained in Ref.[6] and reminded in Tab.2) which maximize the buckling pressure have been used as a reference. In that way, the fitness of the calculated optimized stiffened solution has been evaluated using the relative gain $\eta$ defined as follows:

$$
\eta=\frac{P_{c r}\left([\theta]_{\text {Opti }}^{R}\right)-P_{c r}\left([\theta]_{\text {Opti }}\right)}{P_{c r}\left([\theta]_{\text {Opti }}\right)}
$$

where $P_{c r}\left([\theta]_{O p t i}^{R}\right)$ is the limit of stability for the stiffened composite shell and $P_{c r}\left([\theta]_{O p t i}\right)$ is the critical pressure of the corresponding unstiffened optimized cylinder. This gain has been evaluated both using analytical and FE results: similarly to the study detailed in Ref.[6], 


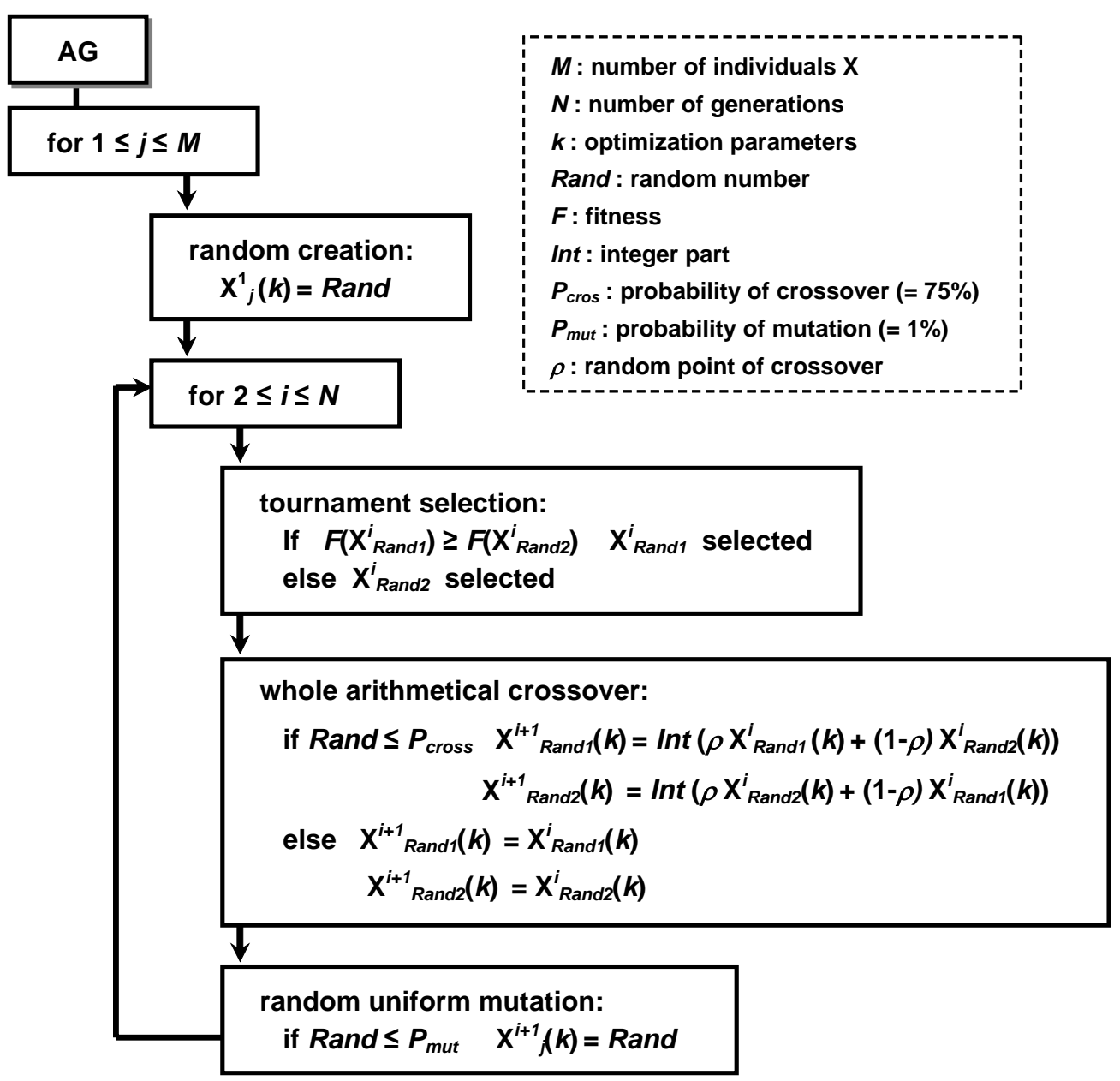

Figure 3: GA flowchart.

\begin{tabular}{llll}
\hline cylinder $^{\circ}$ & {$\left[\boldsymbol{\theta}_{\text {opti }}\right.$} & $\boldsymbol{P}_{\text {cr }}\left([\boldsymbol{\theta}]_{\text {opti }}\right)$ & $(\mathbf{M P a})$ \\
analytical & FEM \\
\hline 1 & & 31.5 & 29.1 \\
2 & {$\left[90_{2} / 60 / 30_{5} / 60 / 90\right]$} & 81.4 & 82.1 \\
3 & {$\left[90_{3} / 75 / 45 / 30_{8} / 60 / 90_{2}\right]$} & 247.3 & 218.6 \\
\hline
\end{tabular}

Table 2: Optimized unstiffened cylinders.

a FE model using Mindlin laminated shell and beam elements has been also computed on Ansys software. The buckling pressures of the $[\theta]_{\text {Opti }}$ reference cylinders are also detailed in Tab.2.

\subsection{Optimization of the stacking sequences}

In this first approach, the stiffeners characteristics (numbers and geometries) and the number of composite plies are fixed. They are assumed to have equal square cross-sections $h_{L} \times h_{L}$ $=h_{C} \times h_{C}$ (see Fig.2) with prescribed side lengths done in Tab.3. The optimal design problem therefore consists to find the optimal laminations $[\theta]^{R}$ opti maximizing the buckling pressure. The results obtained show very small influence of the longitudinal stiffeners a contrary to the rings. Moreover, it should be noticed that the optimized stacking sequences always exhibit a 
$\left[90_{N 1} / \Psi_{1} / \pm 30_{N 2} / \Psi_{2} / 90_{N 3}\right]$ pattern increasing the circumferential rigidity of the shell [5]. The optimized results obtained for the cylinder $n^{\circ} 2$ (and the corresponding gains calculated using the analytical model) are detailed in Tab.4.

\begin{tabular}{llll}
\hline cylinder $^{\circ}$ & 1 & 2 & 3 \\
\hline $\boldsymbol{h}_{\boldsymbol{L}}, \boldsymbol{h}_{\boldsymbol{C}}$ & 5 & 8 & 12 \\
\hline
\end{tabular}

Table 3: Stiffener cross-section dimensions (mm).

\begin{tabular}{llll}
\hline $\boldsymbol{N}_{\boldsymbol{C}}$ & $\boldsymbol{N}_{\boldsymbol{L}}$ & {$[\boldsymbol{\theta}]^{\boldsymbol{R}}{ }_{\text {Opti }}$} & $\eta \mathbf{( \% )}$ \\
\hline 1 & 0 & {$\left[90_{3} / 75 / 30_{9} / 75 / 90_{2}\right]$} & 10.4 \\
2 & 0 & {$\left[90_{3} / 60 / 30_{9} / 90_{3}\right]$} & 20.3 \\
3 & 0 & {$\left[90_{3} / 45 / 30_{8} / 45 / 90_{3}\right]$} & 29.6 \\
4 & 0 & {$\left[90_{3} / 60 / 30_{8} / 75 / 90_{3}\right]$} & 39.1 \\
5 & 0 & {$\left[90_{3} / 60 / 30_{8} / 90_{4}\right]$} & 48.3 \\
0 & 1 & {$\left[90_{4} / 45 / 30_{8} / 60 / 90_{2}\right]$} & 0.7 \\
0 & 2 & {$\left[90_{3} / 75 / 45 / 30_{8} / 75 / 90_{2}\right]$} & 0.9 \\
0 & 3 & {$\left[90_{3} / 75 / 45 / 30_{8} / 75 / 90_{2}\right]$} & 1.1 \\
0 & 4 & {$\left[90_{3} / 75 / 45 / 60 / 30_{8} / 75 / 90_{2}\right]$} & 1.2 \\
\hline
\end{tabular}

Table 4: Optimized laminations of cylinder $n^{\circ} 2$.

For each cylinder, the best optimized solutions have been checked using the FE model as detailed in Tab.5. As observed previously in Ref.[6], the analytical model overestimate the gains. Nevertheless, the FE results have confirmed the stability limit increases due to circumferential stiffeners and optimized laminations.

\begin{tabular}{llllll}
\hline cylinder $^{\circ}$ & $\boldsymbol{N}_{\boldsymbol{C}}$ & $\boldsymbol{N}_{\boldsymbol{L}}$ & $\boldsymbol{[ \theta}^{\boldsymbol{R}}{ }_{\text {opti }}$ & \multicolumn{2}{c}{$\boldsymbol{\eta}$ (\%) } \\
& & & & analytical & FEM \\
\hline 1 & 3 & 0 & {$\left[90_{2} / 30_{7} / 90\right]$} & 16.8 & 16.2 \\
2 & 3 & 0 & {$\left[90_{3} / 45 / 30_{8} / 45 / 90_{3}\right]$} & 29.6 & 16.2 \\
3 & 3 & 0 & {$\left[90_{5} / 75 / 60_{2} / 45 / 30_{8} / 90_{8}\right]$} & 47.1 & 15.8 \\
\hline
\end{tabular}

Table 5: Examples of optimized solutions.

\subsection{Multi-parameter optimization}

In this paragraph, the optimization objective consists in the simultaneous determination of the best lamina stacking sequence and stiffener characteristics. The optimization constraint is to use fixed material volumes equal to those of the corresponding unstiffened cylinders (see Tab.1). The circumferential and longitudinal stiffeners have square $h_{C} \times h_{C}$ and $h_{L} \times h_{L}$ crosssections. The optimization problem can then be formulated as follows:

$$
\begin{aligned}
& \text { find } N,[\theta]^{R}{ }_{\text {Opti }}, N_{C}, h_{C}, N_{L}, h_{L} \\
& \text { leading to a material volume } V_{c} \\
& \text { and maximizing } P_{c r}
\end{aligned}
$$

The optimized results are depicted in Tab.6. For each optimization calculus performed, the number of stringers $N_{L}$ obtained has always been zero. It could be noticed that the lamination pattern of the cylinder $n^{\circ} 1$ is not exactly preserved due to its low number of composite plies. Nevertheless, the reinforcement solutions obtained tend to maximize the circumferential stiffness of the vessels as mentioned previously. 


\begin{tabular}{lllllll}
\hline cylinder $^{\circ}$ & $\boldsymbol{N}_{\boldsymbol{C}}$ & $\boldsymbol{h}_{\boldsymbol{C}}(\mathbf{m m})$ & $\boldsymbol{N}$ & {$[\boldsymbol{\theta}]^{\boldsymbol{R}}{ }_{\text {Opti }}$} & \multicolumn{2}{c}{$\boldsymbol{\eta}(\mathbf{\% )}$} \\
& & & & & analytical & $\mathbf{F E M}$ \\
\hline 1 & 9 & 5.5 & 9 & {$\left[45 / 30_{7} / 90\right]$} & 29.8 & 11.7 \\
2 & 4 & 8.5 & 15 & {$\left[90_{2} / 75 / 30_{8} / 75 / 90_{3}\right]$} & 29.7 & 16.6 \\
3 & 2 & 12.6 & 25 & {$\left[90_{5} / 75 / 60_{2} / 45 / 30_{8} / 90_{8}\right]$} & 26.0 & 16.7 \\
\hline
\end{tabular}

Table 6: Multi-parameter optimization results.

\section{CONCLUDING REMARKS}

This work devoted to the optimization of stiffened composite underwater hulls subjected to buckling leads to the following concluding remarks:

- As observed for the unstiffened hulls previously studied, the optimized stacking sequences exhibit a $\left[90_{N 1} / \Psi_{1} / \pm 30_{N 2} / \Psi_{2} / 90_{N 3}\right]$ typical pattern.

- In the same way and as observed numerically and experimentally in Refs.[5,6], the circumferential reinforcement plays a major role in the increases of the stability limits for such lengthy hulls: on the contrary of the stringers, the rings provide substantial buckling gains.

- The developed optimization tool allows to guide quickly designers for reinforcement solutions: the time of optimization calculus have appeared to be always less than $10 \mathrm{mn}$ on a standard PC. Moreover, the results obtained have also appeared to be reproducible, demonstrating the robustness of the computed GA procedure.

This study provides a reliable tool for the design of large pressure hulls for underwater applications. This method was used for the prospective study of composite buoyancy cans which maintain risers tension in offshore oil production. Another field of investigation is the design of composite deepsea oil/gas separation tanks. The hull of such devices is mainly a large cylinder (2 3 meters in diameter, 10 meter long) submitted to high internal and external pressure.

\section{REFERENCES}

[1] Vinson J.R., The behavior of shells composed of isotropic and composite materials. Kluwer Academic Pub.,1992.

[2] Graham D., Composite pressure hulls for deep ocean submersibles. Composite Structures, 32, 331-343, 1995.

[3] Davies P., Chauchot P., Composites for marine applications - part 2: underwater structures. Mechanics of composite materials and structures, Kluwer Academic Pub., 249260, 1999.

[4] Tsouvalis N.G., Zafeiratou A.A., Papazoglou V.J., The effect of geometric imperfections on the buckling behaviour of composite laminated cylinders under external hydrostatic pressure. Composites Part B, 34-3, 217-226, 2003.

[5] Messager T., Optimisation d'enceintes sous-marines composites (Optimization of composite submarine hulls). PhD Thesis, Lille University, France, July 2000.

[6] Messager T., Pyrz M., Gineste B., Chauchot P., Optimal laminations of thin underwater composite cylindrical vessels. Composite Structures, 58-4, 529-537, 2002. 
[7] Papazoglou V.J., Tsouvalis N.G., Zaphiratou A.A., Parametric study of small scale cylinders under hydrostatic load: flat rigid end closures. MAST III project MAS3-CT970091, report n STL-073-F-98, National Technical University of Athens, 1998.

[8] Sun G., Optimization of stiffened laminated-composite circular-cylindrical shells for buckling. Composite Structures, 23, 53-60, 1993.

[9] Kallassy A., Marcellin J.L., Optimization of stiffened plates by genetic search. Structural Optimization, 13, 134-141, 1997.

[10] Michalewicz Z., Genetic algorithms + data structures = evolution programs, third, revised and extended edition. Springer Verlag, Berlin, 1996.

[11] Miettinen K., Neittaanmäki P., Mäkelä M.M., Périaux J., Evolutionary Algorithms in Engineering and Computer Science. John Wiley, 1999. 